\title{
153. An approach to 24-hour electrocardiogram monitoring in sheep experimental model
}

\author{
Vilius Kviesulaitis ${ }^{1}$, Tomas Kazakevičius ${ }^{2}$, Vytautas Zabiela ${ }^{3}$, Aras Puodžiukynas ${ }^{4}$ \\ Lithuanian University of Health Sciences, Kaunas, Lithuania \\ ${ }^{1}$ Corresponding author \\ E-mail: ${ }^{1}$ viliuskviesulaitis@yahoo.com, ${ }^{2}$ kazakev@gmail.com, ${ }^{3}$ vytautas.zabiela@kaunoklinikos.lt, \\ ${ }^{4}$ aras.puodziukynas@gmail.com
}

Received 22 March 2018; received in revised form 3 June 2018; accepted 14 June 2018 DOI https://doi.org/10.21595/jme.2018.20028

Check for updates

Copyright $(C 2018$ Vilius Kviesulaitis, et al. This is an open access article distributed under the Creative Commons Attribution License, which permits unrestricted use, distribution, and reproduction in any medium, provided the original work is properly cited.

\begin{abstract}
Sheep could be an excellent research subject for large animal experimental studies because of their heart structure and physiology have a lot similarity to the human. Long term ECG monitoring is a convenient tool for human and possibly veterinary clinical practice and research. Continuous ECG recording can be used to monitor and evaluate body physiological responses in various experimental conditions. Experience on sheep ECG monitoring techniques is sparse and mostly focused on short term ECG recordings. We developed a peculiar technique of 24-hour sheep ECG monitoring based on the available data on large mammal model, where we tried to share some insights and practical solutions working with this type of animals for ECG monitoring by using several commercially available ECG monitors also used for the human practice. Some producer specific hardware and software characteristic that we found important in daily practice were also discussed. In conclusion, sheep as big animal is relatively good object for Holter monitoring experimental studies: ECG monitoring is easily affordable, registered ECG signal is of similar quality as in human clinical practice, which is sufficient for the heart rhythm monitoring and arrhythmia and conduction disorder diagnostics. Additional effort might be needed for 24-hour heart rate variability calculations because manual revision of software ECG signal interpretations is requiring, but short-term calculations can be made easy.
\end{abstract}

Keywords: sheep, electrocardiogram, Holter monitoring, heart rate variability.

\section{Introduction}

Long term electrocardiogram (ECG) monitoring, historically known as Holter monitoring (after inventor Norman J. Holter), is an acknowledged tool for diagnostical, observational and experimental purposes. Holter monitoring is applicable on most mammals ranging from rat to horse. Sheep are considered amongst the most suitable large experimental animals as their heart resembles the human both anatomically and physiologically [1-5]. They are convenient to work with due their moderate weigh, which is similar to the average human, and together with calm nature this makes them more suitable for long term experiments, than other similarly available domestic animals, like porcine or large cattle. But data on long term sheep ECG monitoring and particularly the heart rate variability evaluation is sparse and is important yet. During our scientific practice we performed over 200 Holter monitoring's on sheep experimental model [6]. While short duration ECG is never a problem in any anesthetised or immobilised animal, but long-term ECG monitoring in big conscious animal can be challenging. In literature practically, there are no neat solutions to this problem, so we started with knowledge based on human practice and on other mammal ECG recordings which mostly focused on short term $(5 \mathrm{~min}$.) ECG monitoring $[1,5,7-9]$. This sometimes led to excessive measures and precautions prolonging the procedure and sometimes to unexpected flaws resulting in failed record or loss of equipment. In this study we want to represent and discuss our practical experience on repetitive 24-hour ECG monitoring approach using in sheep. 


\section{Animal choice and handling}

ECG monitoring was performed on young mature subjects ranging from 6 to 18 months age. It varies from individual to individual, but more younger animals were founded easier to work with. Size and strength are crucial in terms of animal management, but mental background is also important. Lambs are more curious, cooperative and seem to experience less stress on repetitive monitoring. Whenever possible taming and luring with food is preferable to chasing and catching. In our case one hand fed lamb was almost willing to participate. A shepherd's crook is a handy tool if you manage a big herd. Works best, when the person animals are used to (usually a farmer) participates in all the tasks. It also helps to separate the experimental animals from the maternal herd some hours or the evening before the procedure.

Even if generally calm in nature, sheep are curious, so they are best kept in separate cages during monitoring. Even the breast-fed lambs try to snatch the parts of the equipment tangled under their mother so best is to keep the experimental animals separate from the herd. Walls of the cage should be over 1 meter in height with vertical fence bars, otherwise they will be climbed with all the possible consequences. Individuals who participate in the same process seem to mind their own business, and in cases when we had to place two monitored animals in one cage we had no incidents. But we strongly advise preventing the animal with a monitor on to roam free with the rest of the herd.

\section{Animal preparation}

Signal quality of any ECG recording crucially depends on electrode position and the contact with the skin. Even in human practice some cases can be challenging for an adequate long-term electrode fixation especially in sweaty, hairy individuals. In veterinary practice it gets even more challenging and sheep are not the easiest case model.

Most sheep are well handled by 2 persons when the process gets familiar. But 3 is an optimal number. In the beginning we universally administered short term sedatives to the animals before the procedure. In long term that proved unnecessary and even undesirable. Sedation might be useful only if one intends to do a short ECG record, but even in that case you need to catch and restrain the animal. During a long-term monitoring sedation usually prolongs the procedure which is not a desirable effect in multiple repetitive tasks. It takes time to wait for the effect of the sedative and animals need to be restrained until medication takes over. Sedation of multiple animals at once gives no advantage as they all need to be tended to. Also, it is not always easy to select the adequate dose for the variable duration of the procedure. That sometimes leads to additional doses and prolonged effect, which in turn prolongs the nursing after application of the recorder. In the end, we handled most of the animals without sedation well except maybe the large uncastrated males. General advice is to avoid unruly individuals for the experiment. Uncastrated lambs grow large and hard to handle if you plan a long term follow up (6-12 months) and some individuals are simply to lively. In smaller farms owner can help to identify the desirable qualities of the animals.

Preparing the skin for the electrodes is probably the most time-consuming part of the procedure. Axillar pits are relatively wool free and spacy enough to place the electrodes for short recordings, but fixation for longer periods can be difficult there. To alleviate the procedure animals should be sheared before the procedure. It is best made a day before the recording to shorten the procedure and stress impact on the recording. Shorter wool facilitates the fixation of the electrodes and in most cases, saves time. It also removes most dirt which composed with lanolin and humidity makes fixation of adhesive pad electrodes difficult. Clean and dry is always advised. Shearing is best done with professional electrical shears. It might be worth noting that short wool not always comes handy. It makes handling uncooperative individuals harder. And there is no real necessity to shear the whole animal. A circumferential stripe of some $15-20 \mathrm{~cm}$ width, just caudal from the anterior extremities is enough. It is usually enough to shear and moisture the wool for ECG 
recording with crocodile electrodes but for long term monitoring we recommend the adhesive pad electrodes. For that reason, we strongly advise shaving the skin of remaining wool. Soap or shaving foam facilitate the process. Cut throat razor was the best option as a shaving tool for us. And animal is best laid on a side when doing that.

\section{Electrode placement}

All the monitors we used had 2 channels, and in total 4-5 electrodes. For best short-term ECG signal in literature are recommendations to place electrodes in sagittal axis or use of enhanced limb derivations [10-12]. Frontal plane placement is less preferable due to the position of the sheep heart. But it is more relevant for long term monitoring as it is easier to achieve the electrode stability here. We placed the electrodes slightly caudally $(5-10 \mathrm{~cm})$ from the frontal extremities, in a slightly ventral (precordial) position. Similarly, to the technique used by other authors for the cattle and dog Holter monitoring $[13,14]$. A scheme of electrode placing is shown in Fig. 1.

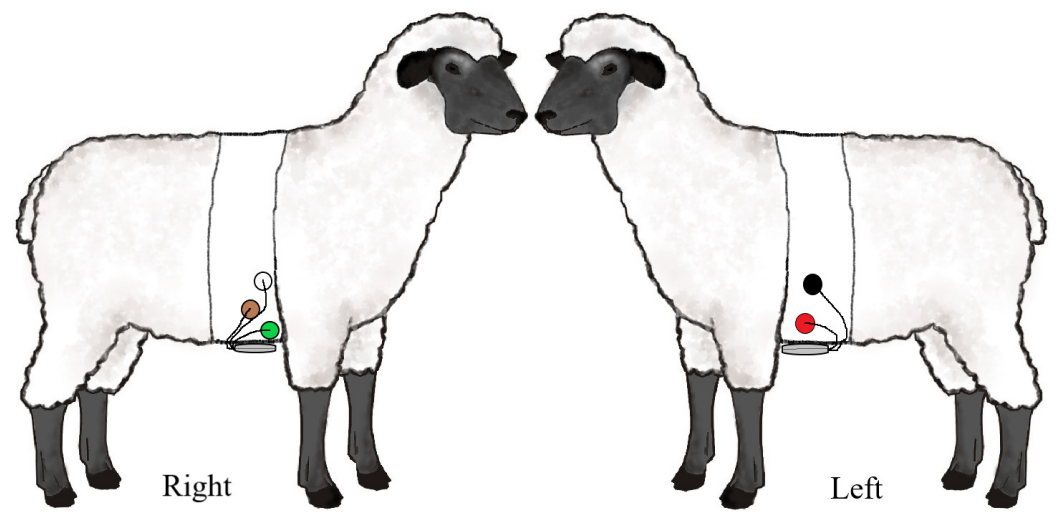

Fig. 1. Precordial lead placement. RED and BLACK electrodes are placed on the left side and WHITE, BROWN and GREEN on the right

We tried three types of the electrodes used in human practice: alligator type, intracutaneous electrodes and self-adherent pad argentum chloride electrodes. We also tried to apply suction electrodes just to check if they might work, but as expected it was with no success. Because of unacceptable probability of infection, we did not consider implanting permanent subcutaneous electrodes. Both alligator and intracutaneous vires were optimal for short term monitoring and provided good ECG signal in immobilised animals. Alligator type was superior because of reusability, ease of application and price. Initially we thought these electrodes would be good for long term monitoring too, but both types performed poorly. Both caused visible irritation of the application site and were hard to maintain in place for 24 hours, especially the alligator type. Animals were obviously trying to scratch and gnaw the application sites which resulted in poor quality and failed recordings. Stressed out animals were resisting subsequent procedures.

Next, which we tried was an adhesive pad electrode. It reproduced a good ECG signal, and clip fixation made it simple to connect to the ECG monitor without any additional modifications unlike the other electrode types. But it was problematic to keep them in place for a long time. Even if the skin was clean shaved and degreased with various types of solvents the pads did not adhere for long and tended to migrate and detach during the monitoring. But initial disappointment led to a solution to glue the electrode pads which was very effective. At first, we used surgical glue but later substituted it with regular 'super' glue for the same effect and a better price. Glue not only facilitated the procedure but made it possible. In more complicated cases animal skin can be additionally prepared with initial application of glue, but usually it is sufficient to glue the margins of the electrode pads (except the electrode itself). Pads can be stuck immediately after the preparation of the skin even if the skin is still wet, which is useful if you prepare the animal laid 
on one side. This way you do not need to roll over it avoiding the dirt sticking to the site of preparation. We did not observe any irritation of the skin and removal of the electrodes was a simple effort. Sometimes signal quality was good enough for a second day of recording. In cases where the electrode pad would stick to firm, it just needs couple days for it to catch some moisture and lanolin to remove easily.

Size of the electrode has some significance too. We found $30 \mathrm{~mm}$ diameter electrode pads the most suitable. Where was no sense to use larger pads as they do not improve the signal quality nor adherence and require clearing additional space, which prolongs the procedure especially if you need to reallocate the electrode.

Gluing the electrode is not enough and it needs additional protection and support. Initially we tried various techniques including improvised harness and rubber belts for multiple reuse. Both did not prove effective enough. Harness got dirty and needed maintenance after a single use. Some different materials than a cotton fabric might work better than it did for us. But still, it is cumbersome to make, and animals are of different size, so several harnesses are needed. Rubber belt was not as convenient as expected too. It was hard to adjust to the conical shape of the sheep chest. It was hard to adjust desirable pressure to the electrodes and belt tended to slide off the electrodes or even dislocate them or pressed the chest too much and caused discomfort to the animal.

Finally, medical plaster tapes of $10-15 \mathrm{~cm}$ width proved to be the most suitable for the electrode support to the chest. The wider tape the better, as it is needed less circles to cover the electrodes which not always lined up in a narrow line. We plastered the monitor along with the electrodes and leads too. Additionally, we used some extra wrapping for the monitor, to avoid plaster adherent and dirt sticking to it. Usually we used a simple rubber glove or a plastic bag. Plaster does not adhere well to the wool, but it does to itself. So, two layers of plaster are needed to achieve the stability and desired support. A second circular layer of continuous plaster holds firm enough to sustain for 24 hours and is usually enough. It also adjusts to any shape of the chest. Additional taping can be made crossing the chest between the legs for further support. Plaster with some elastic properties is the best. If excess humidity is expected an additional layer of a duct tape can be applied on top of the plaster. It is well advised to tape the area of around $10 \mathrm{~cm}$ both sides from the electrodes as the margins of the taping tend to twist and roll slightly which can expose the electrodes.

\section{Monitoring}

A good signal quality for a surface ECG recording is a relatively easy task, but it is much more complicated to maintain the quality for 24 hours. Industry supplies multitude of ECG monitors with varying features that can affect the procedure. When choosing the machine for multiple recordings we think it is important to pay attention to durability, availability of replaceable parts and easy data processing.

We had experience with 3 different devices while monitoring sheep ECG. We do not intend to market the features of any producer, and all the considerations made here are purely practical to point out the desirable features of the Holter monitor for animal experiments. We used Schiller MT-101, SR-Medizinelektronik Cardio Scout recorder, and Custo Cor-3. Schiller MT-101 could be distinguished from the other two by extra-long leads $(80-100 \mathrm{~cm})$. Being able to encircle the whole chest with electrodes is a welcome feature when working with large animals. Both Schiller MT-101 and Custo Cor-3 came with larger hardware box (almost 3 times the size of Cardio Scout) and a glass screen, and interface buttons, which needed more protection from physical damage and were prone to accumulate dirt during multiple reuse. Cardio Scout on the other hand had short leads $(30 \mathrm{~cm})$ but a small and durable box, without any other features on it. Instead it had a Bluetooth connection to a software station (laptop computer) with approximate signal coverage of at least $20 \mathrm{~m}$ which was a notable improvement in means of comfort and durability. It was not a necessary feature in our case, but machine also had an option to monitor several devices online at 
once, which has many clinical and research implications. Attention should be paid to the durability features of the apparatus when choosing machines for multiple 24-hour recordings on large animals. As the animals are prone to dispose the devices when unsupervised. And spare parts should be ready to replace. We lost 2 devices (something like 1 per 100 monitoring) and many more leads during the trial.

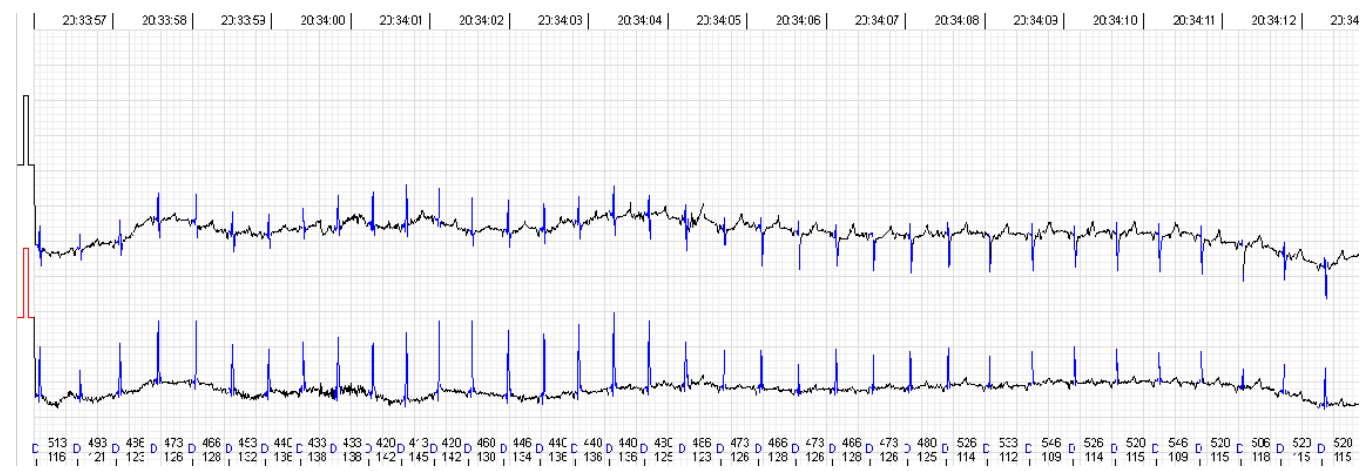

Fig. 2. QRS morphology and voltage shift

Recording a good quality signal is possible in most cases. Automated analysis provides sufficient record quality to evaluate rhythm or conduction disorders. But there are some specific issues while evaluating heart rate variability, which is more artefact sensitive, especially long-term calculations. Sheep QRS complex is relatively small in comparison with a relatively large T wave. In addition, QRS voltage and morphology tends to variate during the record (Fig. 2), especially if registered in frontal plane which seems to be species specific $[9,10,11,15]$. While the ECG waves are easily identifiable visually, automated analysis adopted for human practice is struggling to distinguish between QRS and T wave. Similar situation was described in case of rat ECG monitoring [5]. It mostly leads to 3 types of automated analysis errors: a) T wave identified as QRS, b) T wave identified as premature ventricular contractions and c) missed QRS due to the small voltage (Fig. 3). We found all the types of errors present in all three tested devices, so it does not look like they were software related. Usually, first type of the error can be easily dealt with or even left intact, cause T-T intervals don not really differ from R-R but it surely can affect the 24-hour calculations. Second type of the error can also be solved semi automatically by deleting all the miscalled $\mathrm{T}$ waves. But these errors sometimes come together and require manual correction complex by complex which greatly increases human resource consumption. The 'missing QRS' error was probably the most persistent and irritating. It was repetitive in same animals but not constant. Small QRS voltage seems to be the cause in most cases. Some animals were especially prone to QRS and T wave voltage variation during the monitoring. This was independent of electrode placement. The QRS complexes were easily identifiable by eye but neglected by the software and needed manual correction. When it came to that, Schiller MT-101 somewhat had the most user-friendly interface and tools to offer in our opinion. Simply, less clicks are needed to mark a missing beat or delete one than with other software. And keyboard short keys with other functional solutions alleviate the navigation and processing greatly. This functionality makes a great difference when one needs to mark half an hour of missed beats.

All the mentioned software producers include some basic heart rate variability calculation options. SR-Medizinelektronik Cardio Scout has an option to export the RR intervals for advanced processing with more sophisticated software like Kubios HRV. Which have much stronger tools for automated record quality processing and parameter calculations. This feature might be helpful if higher precision and more complex heart rate variability parameters need to be calculated. 
153. AN APPROACH TO 24-HOUR ELECTROCARDIOGRAM MONITORING IN SHEEP EXPERIMENTAL MODEL. VILIUS KVIESULAITIS, TOMAS KAZAKEVIČIUS, VyTAUTAS ZABIELA, ARAS PUODŽIUKYNAS
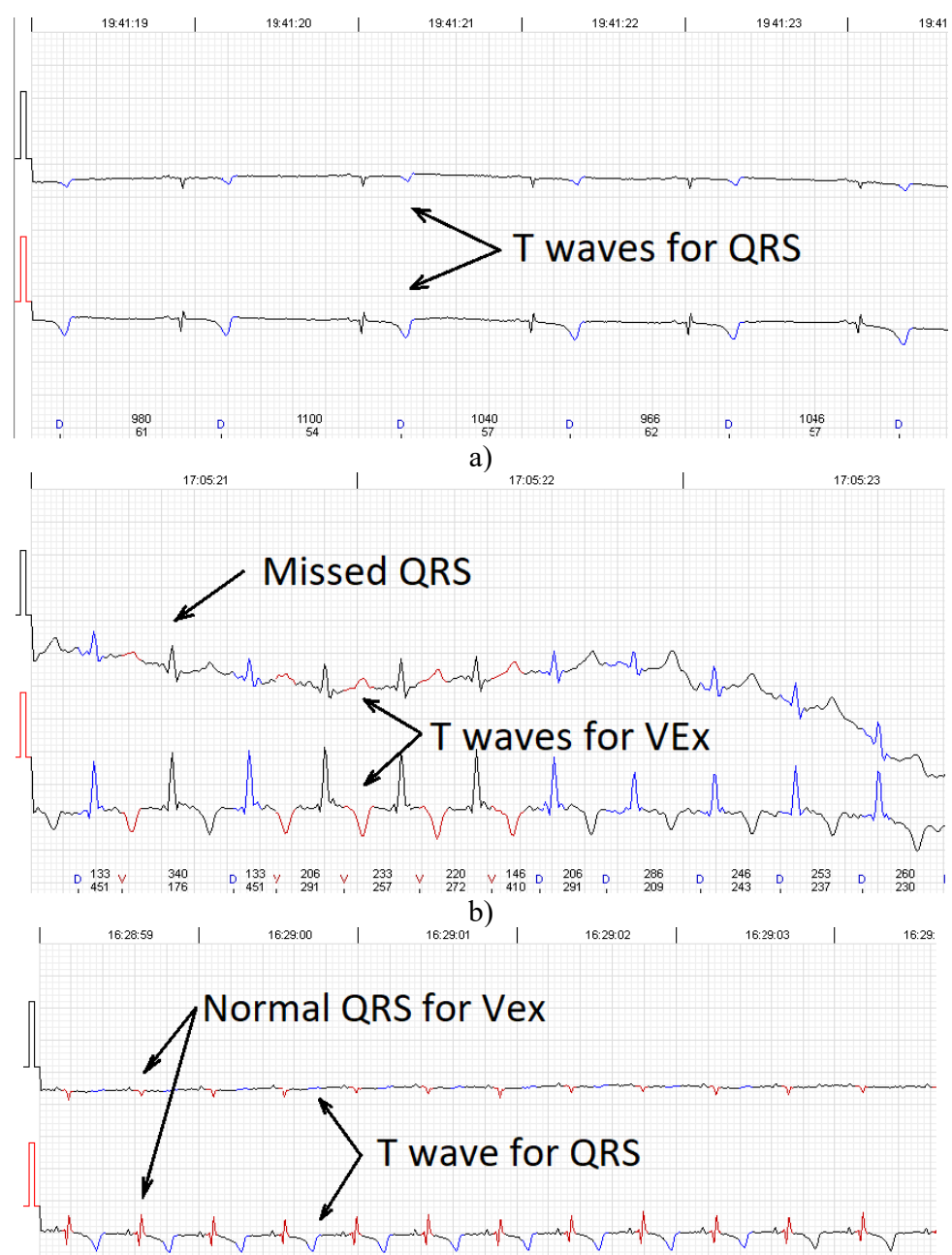

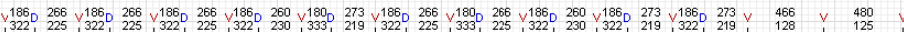
c)

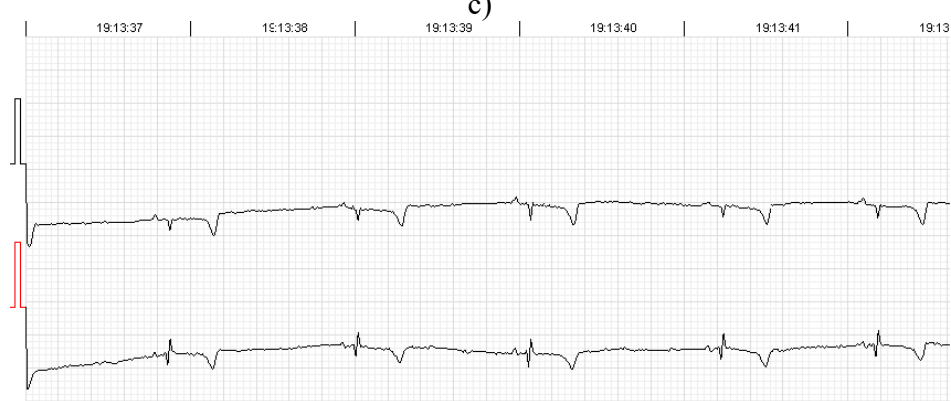

d)

Fig. 3. Errors of the automated analysis leading to false arrhytmia or heart rate variability parameter calculations if not corrected manually. a) Demonstrates a case of rather low wave amplitudes leading to relatively 'deep' $\mathrm{T}$ wave being considered as a QRS complex, marked as 'D' (defined event);

b) shows a case of a single unidentified QRS complex and several $\mathrm{T}$ waves marked as ventricular extrasystole (VEx), marked as ' $\mathrm{V}$ ' (ventricular event); c) a combination of errors where normal QRS complexes are defined as VEx and T waves defined as QRS;

d) software failed to detect a sequence of several low amplitude QRS complexes 


\section{Conclusions}

With appropriate preparation and some basic tools sheep are relatively easy and good experimental model to perform a 24-hour ECG monitoring. ECG signal of similar quality as in human practice can be achieved which is sufficient for the heart rhythm monitoring and arrhythmia and conduction disorder diagnostic. Human ECG monitoring devices are relevant for sheep monitoring. Sufficient signal quality can be achieved for the heart rate variability calculations. This makes 24-hour ECG monitoring a good tool for the large animal experiments as base heart rate values along with the heart rate variability can be used as physiological markers for a wide spectrum of experimental conditions. Additional manual and automated processing is required in some cases.

\section{References}

[1] Ebhart Von Borel, et al. Heart rate variability as a measure of autonomic regulation of cardiac activity for assessing stress and welfare in farm animals - a review. Physiology and Behavior, Vol. 92, 2007, p. 293-316.

[2] Segar J. L. Ontogeny of the arterial and cardiopulmonary baroreflex during fetal and postnatal life. American Journal of Physiology-Heart and Circulatory Physiology, Vol. 273, 1997, p. 457-471.

[3] Woods Jr J. R., Danadavino A., Murayama K., Brinkman C. R., Assali N. S. Autonomic control if cardiovascular functions during neonatal development in adult sheep. Circulation Research, Vol. 40, 1977, p. 401-407.

[4] Kimura Y., Okamura K., Watanabe T., Murotsuki J., Suzuki T., Yano M., et al. Power spectral analysis for autonomic influences in heart rate and blood pressure variability in fetal lambs. American Journal of Physiology-Heart and Circulatory Physiology, Vol. 271, 1996, p. 1333-1339.

[5] Scheer P., Svoboda P., Sepsi M., Janeckova K, Doubek J. The electrocardiographic Holter monitoring in experimental veterinary practice. Physiological Research, Vol. 59, 2010, p. 59-64.

[6] Kviesulaitis V., Puodziukynas A., Pauza D. H., Zabiela V., Kazakevicius T., Vaitkevicius R., Diržinauskas E., Semaška V., Strazdas A., Unikaite R., Rysevaite K., Pauziene N., Zaliunas R. Heart rate variability after radiofrequency ablation of epicardial ganglionated plexuses on the ovine left atrium. BMC Cardiovascular Disorders, Vol. 17, Issue 1, 2017, p. 292.

[7] Reefmann N., et al. Physiological expression of emotional reactions in sheep. Physiology and Behavior, Vol. 98, 2009, p. 235-241.

[8] Konold Timm, Bone Gemma E. Heart rate variability analysis in sheep affected by transmissible spongiform encephalopathies. BMC Research Notes, Vol. 4, 2011, p. 539.

[9] Torío R., Cano M., Montes A., Prieto F., Benedito J. L. Comparison of two methods for electrocardiographic analysis in Gallega sheep. Small Ruminant Research, Vol. 24, 1997, p. 239-246.

[10] Koether K., et al. The normal electrocardiograms in the conscious newborn lambs in neonatal period and its progression. BMC Physiology, Vol. 16, 2016, https://doi.org/10.1186/s12899-016-0020-5.

[11] Rezakhani A., Ejtehadi M. Some electrocardiographic parameters of the fat tailed sheep. Zentralblatt für Veterinärmedizin Reihe A, Vol. 27, Issue 2, 1980, p. 152-156.

[12] Mir S. A., Nazki A. R., Raina R. Comparative electrocardiographic studies and differing effects of pentazocine on ECG, heart and respiratory rates in young sheep and goats. Small Ruminant Research, Vol. 37, 2000, p. 13-7.

[13] Frese D. A., et al. Twenty-four hour Holter monitoring in finishing cattle housed outdoors. Journal of Veterinary Cardiology, Vol. 19, 2017, p. 153-159.

[14] Jean Paul Petrie Practical application of Holter monitoring in dogs and cats. Clinical Techniques in Small Animal Practice, Vol. 20, 2005, p. 173-181.

[15] Jafrin Ara Ahmed, Sagar Sanyal Electrocardiographic studies in goral sheep and black Bengal goats. Research Journal of Cardiology, Vol. 1, 2008, p. 1-8. 